\title{
Sparsity-Aided Low-Implementation cost based On-Board beamforming Design for High Throughput Satellite Systems
}

\author{
Ashok Bandi $^{\dagger}$, Vahid Joroughi ${ }^{\dagger}$, Bhavani Shankar Mysore $\mathrm{R}^{\dagger}$, Joel Grotz ${ }^{*}$ and Björn Ottersten ${ }^{\dagger}$ \\ ${ }^{\dagger}$ Interdisciplinary Centre for Security, Reliability and Trust (SnT), the University of Luxembourg, Luxembourg. \\ *SES S.A., Betzdolf, Luxembourg. \\ Email: \{ashok.bandi, vahid.joroughi, bhavani.shankar, bjorn.ottersten\}@uni.lu, joel.grotz@ses.com
}

\begin{abstract}
Soaring demand for high data rate services entails high throughput satellite (HTS) systems with multi beam architecture, and full frequency and time resources reuse. However, interference among simultaneously served users is the primitive factor that is needed to be addressed before enacting HTS system with this architecture. beamforming has been proposed as a potential technique to mitigate the interference in the literature. Different types of beamforming techniques proposed including beamforming at payload (on-board), beamforming at gateway and hybrid beamforming. On-board beamforming prevails over other techniques due to its advantages-channel information at payload is more recent than gateway and sharing overhead of channel and symbols across multiple gateways is reduced in a multigateway architecture to name a few. Despite these advantages, beamforming at gateway is usually preferred due to the heavy processing cost incurred in beamforming. beamforming processing cost can be split into two factors: design cost and implementation cost. While design cost accounts for the cost involved in design of beamformer, implementation cost accounts for multiplications and additions involved in applying calculated beamformer coefficients to data symbols. Through our study, we noticed that the major contributing factor to processing cost is the implementation cost which accumulates for every data symbol rather than design cost which is incurred only once per channel coherence time which usually relatively longer than many data symbols. Furthermore, implementation cost is dominated by the multiplications involved. Hence, in this work, we address the issue of implementation cost from the perspective of on-board multiplications. We formulate the problem of minimizing on-board implementation cost (multiplications) of a beamformer as a second order cone programming problem with help of $\ell_{1}$ norm constraint on the beamforming matrix subjected to minimum signal-to-interference-noise ratio of simultaneously users and classical total power constraint. We show the efficacy our algorithm over the traditional power minimization method through Monte-Carlo simulations.

Index Terms-High throughout satellite systems, interference,
\end{abstract} beamforming and second order cone programming

\section{INTRODUCTION}

\section{A. Motivation}

In the recent decade, satellite communication utilization has become wide spread and ubiquitous throughout the countries for diverse applications like high data rate connectivity aiming

This work is supported in part by luxembourgish national fund FNR projects PROSAT and SATSENT. at exploiting the unique capabilities in terms of coverage and outreach. This demand for high data rate connectivity pushes the fixed satellite service providers towards employing high throughput satellites where multiple spot beams (multibeam) are employed by applying fractional frequency reuse among beams, leading to provide higher spectral efficiency [1]- [2]. However, one of the major obstacle to deploy multibeam architectures is how to deal with interference which creates among adjacent beams due to the side lobes of the radiation pattern of beams on the Earth surface. To tackle this problem, a preliminary approach would be operating adjacent beams on different frequency bands. In this context, $N_{c}$ is the essential parameter which corresponds to the number of disjoint frequency bands employed on the coverage area $\left(N_{c} \geq 1\right)$. Another promising technique is to reuse the same frequency resources within adjacent beams $\left(N_{c}=1\right)$ by resorting to interference mitigation techniques in order to equalize interbeam interference. In this way, interference mitigation techniques as beamforming in the forward link and multi-user detection in the return link have been proposed in the past studies [3], [7]. Note that, the quality of beamforming/multiuser detecting is sensitive to the quality of Channel State Information (CSI) at the transmitting segment.

Apart from the already mentioned interference limitation, another major issue of multibeam systems is to deal with the large spectral demands on the feeder link, i.e. the bidirectional link between satellite and the Gateway (GW), whose bandwidth requirements increase as it aggregates the traffic of all users. Keeping a full frequency reuse allocation $\left(N_{c}=1\right)$, the required feeder link resources can be calculated as

$$
\mathrm{B}_{\text {feeder-link }}=N B_{\text {beam }},
$$

where $N$ is the number of on-board feed signals. The notations $\mathrm{B}_{\text {beam }}$ and $\mathrm{B}_{\text {feeder-link }}$ are the per-beam and the feeder link required bandwidths, respectively. From (1), it is evident that any beam available bandwidth enhancement forces the feeder link resources to be increased accordingly and, eventually the feeder link might become the communication bottleneck.

In the context of applying interference mitigation techniques and optimizing feeder link resources in multibeam networks, the following possible configurations can be conceived: 
1) Ground Processing (GP): single GW employs an interference mitigation technique to cope with increased level of interbeam interference. Satellite payload works in the transparent mode. There is no certain feeder link optimization strategy such that a set of $\mathrm{B}_{\text {feeder-link }}$ in (1) is required [3].

2) Hybrid Space-Ground Processing (HSGP): the ground segment consists of a single GW that embeds interference mitigation techniques to manage the inter-beam interference. On the other hand, the payload employs an on-board beamforming $(\mathrm{BFN})$ to assign [4] $\mathrm{B}_{\text {feeder-link-onboard }}=K \mathrm{~B}_{\text {beam }}$, where $\mathrm{B}_{\text {feeder-link-onboard }}$ denotes the feeder link resources that is required after employing the on-board BFN with $\mathrm{B}_{\text {feeder-link-onboard }}<\mathrm{B}_{\text {feeder-link }}$ and $N>K$. However, the payload complexity and calibration requirements of any onboard BFN are currently its main drawback.

3) Multi Gateway Processing (MGP): this architecture exploits the multiplexing diversity by reusing all the available feeder link bandwidth across multiple GWs. The GWs employ interference mitigation techniques and the required feeder link bandwidth is optimized with the number of GWs. In this context, the required feeder link bandwidth becomes [5] $\mathrm{B}_{\text {feeder-link-MG }}=\frac{N}{F} \mathrm{~B}_{\text {beam }}$, where $F$ is the number of GWs, and $\mathrm{B}_{\text {feeder-link-MG }}$ denotes the feeder link bandwidth which is required at MGP architecture. Indeed, the MGP architecture reduces the required feeder link bandwidth to $\mathrm{B}_{\text {feeder-link-MG }}<$

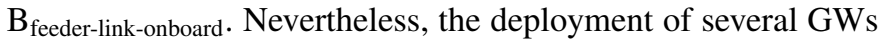
increases the cost of the system.

This study investigates the forward link of a MGP scheme, where a BFN scheme is applied at the payload. The BFN is developed while:

(i) A low complex payload infrastructure is targeted.

(ii) Inter-beam interference shall be mitigated, leading to optimize achievable rate at each user terminal.

Furthermore, we develop a BFN scheme aiming to fulfill the requirements in (i) and (ii). Some additional benefits can be realized via applying BFN in MGP network. First, it is not necessary to establish a CSI feedback mechanism between satellite and the GWs. Second, CSI exchange mechanism is not needed among GWs, leading to a low complex transmitting segment infrastructure. Third, in case of failing one GW, the traffic can be easily rerouted to the satellite through other GWs without applying any extra signal processing schemes at the GWs.

The realization of on-board beamforming entails low complexity beamformer design and low implementation cost. Many iterative and non-iterative low complexity beamformer designs exist in the literature. Design of classical zero-forcing (ZF) and minimize mean square error (MMSE) are two widely used non-iterative beamformers due to their low-complexity in the design. Several convex and non-convex iterative beamformer designs addressing various design aspects of beamforming such as power minimization [8], weighted sum rate (WSR) maximization [9] etc are proposed in the literature. Iterative methods are generally have high complexity than non-iterative methods. However, to the best of our knowledge, design of beamformer with the objective of minimizing implementation cost is not addressed in the literature.

As the satellite operates on signal bandwidth, which is usually of the order of several GHzs, implementation of onboard beamforming in the real-time and power consumed for implementation become pivotal. As mentioned previously, the implementation cost involves multiplication and additions of the beamformer coefficients with on-board frame symbols or sample and it is primarily dominated by multiplications. Although efficient implementation techniques, exist in the literature, aids in reducing multiplication cost, further contribution can be made by avoiding the multiplications to the extent possible. In this work, we address the low-implementation cost of the beamformer by sparsifying the beamformer matrix subject to design constraints.

Following are the contributions made through this paper:

- We formulate the on-board beamforming problem with objective of minimizing implementation subject to minimum rate constraints of users with the help of sparsity constraints. We refer this problem as sparse beamforming problem

- Aforementioned spare beamforming problem contains the $\ell_{0}$ norm of the beamformer as the objective, hence it is a NP-hard in general which requires non-polynomial time complexity algorithm for attaining a global solution. Hence, we relax $\ell_{0}$ norm to $\ell_{1}$ norm, which convexifies the problem.

- We show the efficacy of the proposed design, over the traditional designs with respect to implementation cost, through Monte-carlo simulations.

The rest of the paper is organized as follows. System model is explained in section II and design of on-board precoding and algorithm to solve it is described in section III. Section IV contains the simulation results of the proposed algorithms and section $\mathrm{V}$ contains the conclusions.

Notation: Throughout this paper, the following notation are adopted. Boldface uppercase letters denote matrices and boldface lowercase letters refer to column vectors. (. $)^{H}$ and $(.)^{T}$ a denote Hermitian transpose and transpose matrices, respectively. $\mathbf{I}_{N}$ builds the $N \times N$ identity matrix. $(\mathbf{A})_{i j}$ represents the ( $i$-th, $j$-th) element of matrix $\mathbf{A}$. The notation diag represents a diagonal matrix. $\mathrm{E}\{$.$\} and \|$.$\| refer to the$ expected value operator and the Frobenius norm operators, respectively.

\section{SCENARIO DESCRIPTION}

\section{A. System Model}

Consider the forward link of a MGP multibeam satellite system, where a single geosynchronous (GEO) satellite with multibeam coverage provides fixed broadband services to a large set of users with $N$ feeds and $K$ beams, with $N=K$. By employing a Time Division Multiplexing (TDM) scheme, at each time instant, a total of $K$ single antenna users, i.e. exactly one user per beam, is simultaneously served by a set of $F$ GWs. Without loss of generality, we assume each GW 
has access to an identical number of feeds ${ }^{1}$. In particular, we let $f$-th GW, with $f=1, \ldots, F$, employs $N_{f}=\frac{N}{F}$ on-board feeds to transmits its signals. In addition, it is conceived that each GW transmits a sub set of traffic streams to satellite. Again, without loss of generality, we consider an identical set of traffic streams at each GW. In this context, for available $K$ number of beams, $f$-th GW calculates and transmits a sub set of $K_{f}=\frac{K}{F}$ traffic streams, one stream per user such that $f$-th GW serves a sub set of $K_{f}$ out of $K$ user terminals within the whole coverage area. To make sure that the user demands are met, we further assume an aggressive $N_{c}=1$ frequency reuse where all the beams use the same user link spectrum leading to inter-beam interference among the beams. In consequence, inter-beam interference in user link become the bottleneck of the whole system motivating the use of the interference mitigation techniques.

Remark 1. Even with highly directive antennas the feeder link originating at different GWs are partially interfering. Nevertheless, in this work we assume that GWs are sufficiently separated on the Earth surface and space so that the interfeeder link interference can be ignored. In this context, the received signal at the coverage area can be modeled as

$$
\mathbf{y}=\sqrt{\kappa} \mathbf{H B} \mathbf{x}+\mathbf{n},
$$

where $\mathbf{y}$ is a $K \times 1$ vector containing the symbols received by $K$ users, one per beam, at a given time instant. The $K \times 1$ vector $\mathbf{x}=\left[\mathbf{x}_{1}, \ldots, \mathbf{x}_{f}, \ldots, \mathbf{x}_{F}\right]^{T}$ denotes the stacked transmitted signals at all the on-board feeds with $\mathrm{E}\left\{\mathbf{x x}^{H}\right\}=\mathbf{I}_{K}$. The notation $\mathbf{x}_{f}$ of size $K_{f} \times 1$ is a vector denotes the signals transmitted by $f$-th $\mathrm{GW}$ to the satellite. The vector $\mathbf{n}$ of size $K \times 1$ contains the stacked zero mean unit variance Additive White Gaussian Noise (AWGN) at $K$ users such that $\mathrm{E}\left\{\mathbf{n n}^{H}\right\}=\mathbf{I}_{K}$. The BFN weights are included in matrix $\mathbf{B}$. The scalar $\kappa$ is the power scaling factor and must adapt with

$$
\operatorname{trace}\left(\mathbf{B B}^{H}\right) \leq P
$$

where $P$ is the transmit power of $N$ feeds.

Note that the transmit power constraint in (3) is set considering B. Throughout this paper it is conceived that the power allocation mechanism is located at the array fed reflector system with $N$ embedded feeds.

In the sequel, $\mathbf{H}$ is the overall $K \times N$ user link channel matrix whose element $(\mathbf{H})_{i j}$ represents the gain of the link between the $i$-th user (in the $i$-th beam) and the $j$-th satellite feed. The matrix $\mathbf{H}$ includes the propagation losses and radiation pattern, and as such is decomposed as [5]

$$
\mathbf{H}=\operatorname{diag}\left(\frac{1}{\sqrt{A}_{1}}, \ldots, \frac{1}{\sqrt{A}_{K}}\right) \mathbf{W},
$$

where $A_{k}$ denotes the propagation losses from the satellite to the $k$-th user. $\mathbf{W}$ is a $K \times N$ matrix which models the feed

\footnotetext{
${ }^{1}$ This implies the fact that an identical number of feeder link resources and on-board feeds is assumed so that the on-board BFN directly converts one by one feeder link signals to on-board feed signals.
}

radiation patterns, the path loss and the received antenna gain. The $(k, n)$-th entry of $\mathbf{W}$ is modeled as

$$
(\mathbf{W})_{k n}=\frac{\sqrt{W_{R}} g_{k n}}{4 \pi \frac{d_{k}}{\lambda} \sqrt{k_{B} T_{R} B_{W}}}
$$

where $W_{R}$ denotes the user receive antennas power gain. $g_{k n}$ is referring to the gain (in power) from feed $n$ toward the $k$-th user such that the respective feed transmit gain is $10 \log _{10}\left(\left|(\mathbf{W})_{k n}\right|^{2}\right)$ if expressed in $\mathrm{dBi}$. Finally, $d_{k}$ is the distance between the $k$-th user and the satellite, $\lambda$ the carrier wavelength, $k_{B}$ the Boltzmann constant, $T_{R}$ the receiver noise temperature, and $B_{W}$ the carrier bandwidth.

\section{DESIGN OF ON-BOARD BEAMFORMING}

On-board design of beamforming entails two factors into the design: Low design complexity and low implementation cost. While the design complexity considers the complexity involved in calculation of beamforming matrix, implementation cost considers the cost of implementing beamforming feature with designed beamforming matrix. The dominant factor in the implementation cost is incurred by multiplication of beamforming coefficients with data symbols or samples. Hence, we assume that implementation cost which includes the power and time required to implement beamforming feature on-board can be translated into the order of number of multiplications. Moreover, the number of multiplications can be translated to number of non-zeros in BFN matrix. While efficient implementation techniques help in reduction of complexity, sparsification of BFN (zeroing out beamforming coefficients) helps further in reduction as it reduces number of multiplications. In this work, we address the implementation cost of BFN by sparsifying the beamforming matrix to minimize implementation cost subject to total transmit power and minimum rate constraints.

\section{A. Low implementation cost modeling}

Let $\mathbf{h}_{i}$ be the $i^{\text {th }}$ row of $\mathbf{H}$ and $\mathbf{b}_{i}$ be $i^{\text {th }}$ column of $\mathbf{B}$. With the help of aforementioned definitions, the problem of minimizing the implementation cost subject to total power and minimum SINR constraints can mathematically be formulated as:

$$
\begin{aligned}
\mathcal{P}_{1}: & \min _{\mathbf{B}} \\
\text { subject to } C_{1}: & \frac{\mid \mathbf{B} \|_{0}}{\sigma^{2}+\sum_{j \neq i}^{N}\left|\mathbf{h}_{i}^{H} \mathbf{b}_{j}\right|^{2}} \geq \epsilon_{i}, \forall i \\
C_{2}: & \|\mathbf{B}\|_{2} \leq P_{0}, \forall i,
\end{aligned}
$$

where $P_{0}$ is the available total transmit power, $\|\mathbf{B}\|_{0}$ is the $\ell_{0}$ norm of $\mathbf{B}$ and $\epsilon_{i}$ is the minimum SINR constraint of user $i$.

Remarks:

- The problem $\mathcal{P}_{1}$ is non-convex due to non-convex objective

- $\|\mathbf{B}\|_{0}$ counts number of non-zeros in $\mathbf{B}$. Hence the the objective is also combinatorial and known to be NP-hard for high dimensional matrices. 
- The minimum rate constraint of user $i$, for $i=1, \ldots, N$, in $C_{1}$ in $\mathcal{P}_{1}$ appears to be non-convex. However, constraint $C_{1}$ can be written as a second order cone constraint which is convex.

Obtaining a global solution to $\mathcal{P}_{1}$ entails the exhaustive search over the beamforming space due to the combinatorial nature of the problem (i.e. $\|\mathbf{B}\|_{0}$ ). Exhaustive search based algorithms become non-polynomial time complex even for practically realizable dimensions of B. Many non-combinatorial relaxations of $\mathbf{B}$ are proposed in literature. Relaxations are primarily classified into two categories: Convex and nonconvex. In non-convex relaxations, $\|\mathbf{B}\|_{0}$ is relaxed to $\|\mathbf{B}\|_{p}$ for $0<p<1$. In convex relaxation, $\|\mathbf{B}\|_{0}$ is relaxed to $\|\mathbf{B}\|_{1}$. Under particular conditions, $\|\mathbf{B}\|_{1}$ based relaxation is shown to obtain the same solution as $\|\mathbf{B}\|_{0}[10]$.

In this work, we adopt the $\ell_{1}$ relaxation as it results the convex objective. The problem $\mathcal{P}_{1}$ with convex relaxation of $\|\mathbf{B}\|_{0}$ can be mathematically formulated as

$$
\begin{aligned}
\mathcal{P}_{2}: & \min _{\mathbf{B}} \\
\text { subject to } C_{1}: & \frac{\mid \mathbf{B} \|_{1}}{\sigma^{2}+\sum_{j \neq i}^{N}\left|\mathbf{h}_{i}^{H} \mathbf{b}_{j}\right|^{2}} \geq \epsilon_{i}, \forall i \\
C_{2}: & \|\mathbf{B}\|_{2} \leq P_{0}, \forall i
\end{aligned}
$$

Although, the objective in $P_{2}$ is convex, the problem $P_{2}$ can not be solved efficiently with existing tools, that can solve convex problems efficiently, due to constraint $C_{1}$. The problem $P_{2}$ can be formulated as a convex problem by converting constraint $C_{1}$ in $P_{2}$ as second order cone constraint problem. The constraint $C_{1}$ can rearranged as

$$
\left(1+\frac{1}{\epsilon_{i}}\right)\left|\mathbf{h}_{i}^{H} \mathbf{b}_{i}\right|^{2} \geq\left\|\begin{array}{c}
\sigma \\
\mathbf{h}_{i}^{H} \mathbf{b}_{1} \\
\vdots \\
\mathbf{h}_{i}^{H} \mathbf{b}_{N}
\end{array}\right\|_{2}^{2}, \forall i
$$

Suppose $\mathbf{B}$ is an optimal beamforming matrix for $P_{2}$, then $\mathbf{B} \operatorname{diag}\left\{\mathrm{e}^{j \phi_{i}}\right\}$, where $\phi_{i}$ for $i=1, \ldots, N$ are arbitrary phases, is also optimal. This can be easily verified as the phase does not alter the objective nor the constraints. Hence, we restrict ourselves to the design of the beamformers with $\mathbb{R e}\left\{\mathbf{h}_{i}^{H} \mathbf{b}_{i}\right\} \geq 0, \operatorname{Im}\left\{\mathbf{h}_{i}^{H} \mathbf{b}_{i}\right\}=0, i=1, \ldots, N$ as it helps to reformulate the constraint as a convex constraint. With this newly imposed restriction on real and imaginary parts of $\mathbf{h}_{i}^{H} \mathbf{b}_{i}$, for $i=1, \ldots, N$, the constraint $C_{1}$ in $P_{2}$ is feformuated as

$$
\left(\sqrt{1+\frac{1}{\epsilon_{i}}}\right) \mathbf{h}_{i}^{H} \mathbf{b}_{i} \geq\left\|\begin{array}{c}
\sigma \\
\mathbf{h}_{i}^{H} \mathbf{b}_{1} \\
\vdots \\
\mathbf{h}_{i}^{H} \mathbf{b}_{N}
\end{array}\right\|, \forall i
$$

The problem $P_{2}$ with the convex reformulation of constraint $C_{1}$ is

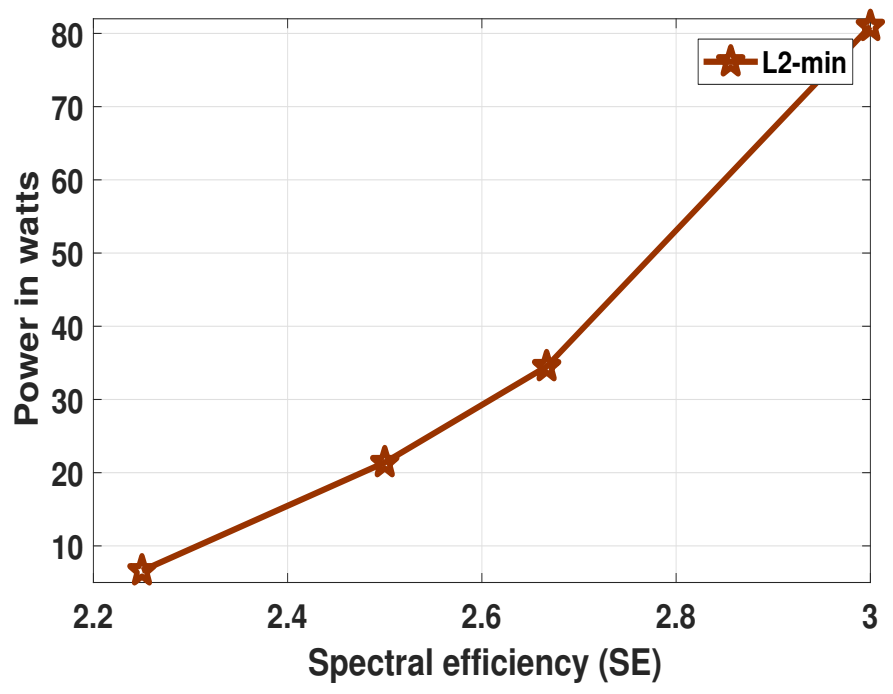

Fig. 1: Power consumed by L2-minimization versus spectral efficiency for 12 beam HTS system with one user per beam

$$
\begin{gathered}
\mathcal{P}_{3}: \min _{\mathbf{B}}\|\mathbf{B}\|_{1} \\
\text { subject to } C_{1}:\left(\sqrt{1+\frac{1}{\epsilon_{i}}}\right) \mathbf{h}_{i}^{H} \mathbf{b}_{i} \geq\left\|\begin{array}{c}
\sigma \\
\mathbf{h}_{i}^{H} \mathbf{b}_{1} \\
\vdots \\
\mathbf{h}_{i}^{H} \mathbf{b}_{N}
\end{array}\right\|, \forall i \\
C_{2}:\|\mathbf{B}\|_{2} \leq P_{0}, \forall i
\end{gathered}
$$

The problem $P_{3}$ is convex problem since the objective and the constraint are convex. Hence, the problem $P_{3}$ can be solved globally and efficiently. Existing tools like CVX can be used to solve the problem $P_{3}$ efficiently. In the next section we present the performance of $P_{3}$ through Monte-Carlo simulations.

\section{NumericAl Results}

\section{A. System setup}

To compare the performance of the proposed scenarios in this study, Monte Carlo simulations have been carried out. The simulation setup is based on an array fed reflector antenna/feed provided by European Space Agency (ESA) in the context of NGW project with $N=K=12$ feeds/beams, at each time instant, which serve a single user per beam and spread over the whole Europe [6]. Results have been averaged for a total of 500 channel realizations. For each beam different user positions is considered at consecutive channel realizations. The detail of simulation parameters are collected in beamforming I. Note that the channel fading statistics corresponds to the city of Rome. We compare the achieved implementation cost for different transmit powers by the problem $P 3$, referred as L1-minimization (L1-min), with classical power minimization problem [8], referred as L2-minimization (L2-min). The classical power minimization problem given in [8] can be obtained replacing $\|\mathbf{B}\|_{1}$ in (10) with $\|\mathbf{B}\|_{2}$ and ignoring constraint $C_{2}$. 


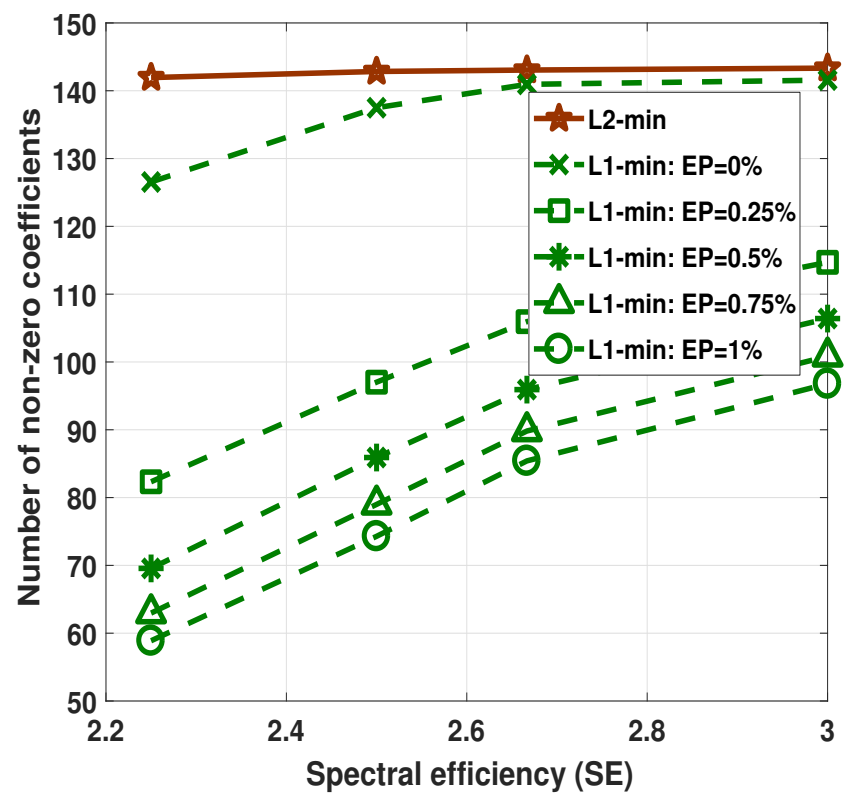

(a) Number non-zero precoder coefficients for L2-min and L1min for different transmission powers versus spectral efficiency

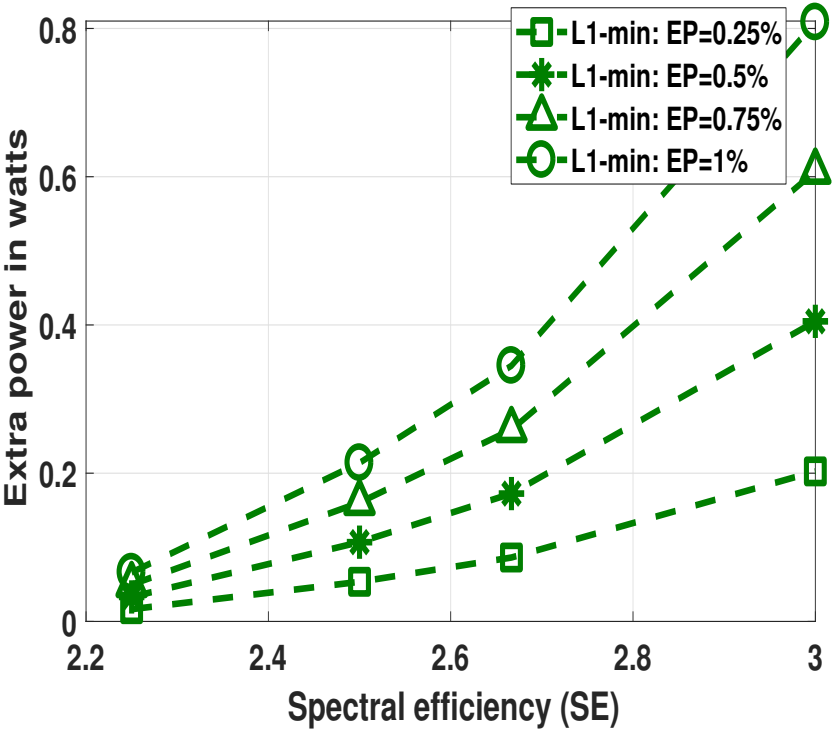

(b) Extra power consumed by L1-minimization versus spectral efficiency

Fig. 2: Performance comparison of L2-min with L1-min for different transmission powers for 12 beam HTS system with one user per beam.

\section{B. Results}

In figure $2 b$, The total transmit required in watts for 4 different spectal efficiencies (SE) $(2.20,2.5,2.666,3 \mathrm{bps})$ is plotted. In figure $2 \mathrm{a}$, we compare the implementation cost of L2-min with L1-min for the previously mentioned SEs. The reduction in the implementation cost of L1-min can be observed that in figure $2 \mathrm{a}$, when it is supplied with small percentage of extra power (EP) than L2-min. For example in figure $2 \mathrm{a}$ for $\mathrm{SE}=2.25 \mathrm{bps} / \mathrm{Hz}$, the number of non-zeros in beamforming matrix of L2-minimization is 144 for the
TABLE I: USER LINK SIMULATION PARAMETERS

\begin{tabular}{|l|l|}
\hline Parameter & Value \\
\hline Satellite height & $35786 \mathrm{~km}$ (GEO) \\
\hline Satellite longitude, latitude & $10^{\circ}$ East, $0^{\circ}$ \\
\hline Frequency & $20 \times 10^{9}$ \\
\hline Earth radius & $6378.137 \mathrm{Km}$ \\
\hline Feed radiation pattern & Provided by ESA [6] \\
\hline Number of feeds N & 12 \\
\hline Number of beams & 12 \\
\hline Carrier frequency & $20 \mathrm{GHz}($ Ka band) \\
\hline Total bandwidth & $500 \mathrm{MHz}$ \\
\hline Atmospheric fading & Rain attenuation [6] \\
\hline Roll-off factor & 0.25 \\
\hline User antenna gain & $41.7 \mathrm{dBi}$ \\
\hline clear sky gain & $17.68 \mathrm{~dB} / \mathrm{K}$ \\
\hline
\end{tabular}

transmit power of $6 \mathrm{~dB}$ but with total transmit power of $6.07 \mathrm{~dB}$ (see figure $2 \mathrm{~b}$ for extra power needed), L1-minimization can achieve the same SE with beamformer matrix which has less than 60 non-zero coefficients. Similarly, it can be observed in figure $2 \mathrm{a}$, the implementation cost that can be achieved with L1-min for different SEs and transmit powers.

In figure $2 b$, we plot the amount extra power needed for different SEs in achieving the implementation costs provided in figure 2a. From figure 2, we can observe that L1-min can achieve only a small gain in the implementation cost if $P_{0}$ is same as optimal power, say $P_{\mathrm{opt}}$, achieved by L2min. However, we can see larger gain in implementation cost of L1-min for $P_{0}$ which is slightly greater than $P_{\text {opt }}$. We also observe that the gain in implementation cost are larger for lower SEs, this because lower SEs can afford to have some interference. As a result, L1-min makes the most of the beamforming coefficients zeros allowing the interference that can be affordable at this low SEs. However, we see the gain in the implementation cost diminishes with increase in SE, this is due to the fact that higher SEs demand the lowest interference also to be canceled hence the L1-min can not make many of the coefficients as it allows the interference that can not be affordable at this high SEs.

\section{CONCLUSion}

In this paper, design of on-board precoding is considered with the objective of minimizing implementation cost subject to minimum SINR requirement of users and total transmission power constraints. The major contribution of the implementation cost is by the multiplications involved in applying the precoder coefficients to the data symbols. Hence, we modeled the objective of minimizing the implementation cost as the objective of minimizing the number of non-zero precoder coefficients as a zero precoder coefficient avoids the need of multiplication. Hence, the minimizing the implementation cost is modelled with the help $\ell_{0}$ norm constraint on precoding matrix. However, the $\ell_{0}$ norm objective problem is NP-hard so we relax objective to $\ell_{1}$ norm which makes the problem convex. Finally, we show the reduction in the implementation cost compared to the classical power minimization problem through Monte-Carlo simulations. 


\section{REFERENCES}

[1] Daniel Minoli, "Innovations in satellite communications technology," in John Wiley \& Sons Inc. Hoboken, USA, 2015.

[2] J.D. Gayrard, 'Terabit satellite: Myth or reality?" in First International Conference on Advances in Satellite and Space Communications (SPACOMM), pp. 1-6, July 2009.

[3] D. Christopoulos, S. Chatzinotas, G. Zheng, J. Grotz, and B. Ottersten, "Linear and nonlinear techniques for multibeam joint processing in satellite communications," in Journal on Wireless Communications and Networking, vol. 2012, no. 1, p. 162, 2012.

[4] V. Joroughi, M. A. Vazquez, A. Ana Perez-Neira and B. Devillers, ”Design of an on board beam generation process for a multibeam broadband satellite system," in IEEE Transactions on Wireless Communications, Vol. 13, pp. 1-14, March 2017.

[5] V. Joroughi, M. A. Vzquez and A. I. Prez-Neira, "beamforming in multigateway multibeam satellite systems," in IEEE Transactions on Wireless Communications, Vol. 15, No. 7, pp. 1-13, July 2016.

[6] Call of Order 2-Task 1, "beamforming with User Clustering," Next Generation Waveforms for Improved Spectral Efficiency, report of ESA contract NO:4000106528/12/NL/NR.

[7] G. Zheng, S. Chatzinotas and B. Ottersten, "Generic optimization of linear beamforming in multibeam satellite systems, in IEEE Transactions on Wireless Communications, vol. 11, no. 6, pp. 2308-2320, 2012.

[8] A. Wiesel, Y. Eldar, and S. Shamai, "Linear beamforming via conic optimization for fixed MIMO receivers, in IEEE Trans. Signal Process., vol. 54, no. 1, pp. 161176, Jan. 2006.

[9] P. C. Weeraddana, M. Codreanu, M. Latva-Aho, A. Ephremides, and F. Carlo, "Weighted Sum-Rate Maximization in Wireless Networks: A Review (Foundations and Trends in Networking), in vol. 6. Hanover, MA, USA: Now, 2012.

[10] Simon Foucart, "A note on guaranteed sparse recovery via 1minimization, in Applied and Computational Harmonic Analysis, Volume 29, Issue 1, 2010, Pages 97-103, ISSN 1063-5203.

11] Thomas Lipp and Stephen Boyd, "Variations and extension of the convex-concave procedure," in http://stanford.edu/ boyd/papers/cvx_ ccv.html, year $=2016$

[12] A. L. Yuille and A. Rangarajan, "The concave-convex procedure (CCCP)," in NIPS, 2001

[13] Gert R. Lanckriet and Sriperumbudur, Bharath K., "On the Convergence of the Concave-Convex Procedure," Advances in Neural Information Processing Systems vol. 22, pp. 1759-1767, 2009.

[14] , Alex J. Smola and S. V. N. Vishwanathan and Thomas Hofmann, "Kernel Methods for Missing Variables", book. IN PROCEEDINGS OF THE TENTH INTERNATIONAL WORKSHOP ON ARTIFICIAL INTELLIGENCE AND STATISTICS, pp. 325-332, 2005

[15] T. Lipp and S. Boyd, Variations and extension of the convexconcave procedure, Optim. Eng., vol. 17, no. 2 pp. 263287, Jun. 2016. 